\title{
Peut-on encore améliorer la protection radiologique dans les mines souterraines ?*
}

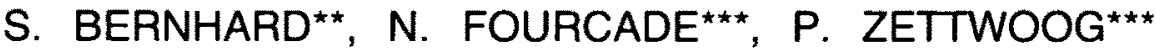

(Manuscrit reçu le 15 janvier 1992)

RÉSUMÉ Après avoir posé le problème, des résultats et commentaires sont donnés pour illustrer les efforts d'optimisation faits par les exploitants miniers. Dans le cycle du nucléaire, les travailleurs les plus exposés, en routine, sont les mineurs d'uranium (moyenne pour les mines souterraines entre 20 et $30 \mathrm{msv}^{-a^{-1}}{ }^{\text {). Dans les mines }}$ d'uranium, les exploitants font, surtout depuis 10 ans, de gros efforts pour diminuer les doses collectives et pour respecter la réglementation en vigueur basée sur les recommandations de la CIPR (publications 26 et 47). Depuis 1988, chez les mineurs français, aucune dose effective (cumul) ne dépasse la limite fondamentale

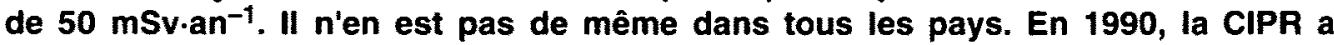
édité de nouvelles recommandations (publications 60 et 61 ) où la limite fondamentale est telle qu'en moyenne elle ne doit pas dépasser $20 \mathrm{mSv} \cdot \mathrm{an}^{-1}$. Pour respecter cette limite, les exploitants devront prendre un certain nombre de dispositions plus ou moins contraignantes. Les moyens financiers supplémentaires seraient importants dans les mines existantes. De nouveaux concepts d'exploitation minière seraient à rechercher_pour_les_mines_nouvelles.-Indépendamment-des-aspects financiers, les dépenses faites pour essayer de réduire un risque déjà très faible (aléatoire et à long terme) ne seraient-elles pas mieux employées pour améliorer les postes de sécurité classique ? On voit, de plus, apparaître toute une série d'effets pervers allant à l'encontre du but recherché. En conclusion, l'amélioration de la protection radiologique peut être obtenue plus efficacement par la mise en cuvre du principe de l'optimisation de la dose collective que par un accroissement de la sévérité des limites de dose individuelles.

ABSTRACT The efforts of optimization made by mine operators to improve radiation protection are illustrated by results and comments. Routinely, uranium miners are the most exposed workers in the fuel cycle (average : 20-30 $\mathrm{mSv} \cdot \mathrm{y}^{-1}$ for underground mines). In uranium mines, especially for 10 years, operators have been striving to lower collective doses and comply with the regulation in force based on ICRP recommendations. Since 1988, french uranium miners have registered no effective cumulated doses exceeding the basic limit of $50 \mathrm{msv} \cdot \mathrm{y}^{-1}$, which does not occur in every country. In 1990, ICRP issued new recommendations lowering the basic limit so that the average should not exceed $20 \mathrm{mSv} \cdot \mathrm{y}^{-1}$. To comply with the limit, the operators should make a number of more or less restricting arrangements. Additional financial means would become important in present mines. New operating concepts should be sought for new mines. Independently of the financial aspects, should not the expenses made towards lowering a risk already very low (hypothetic and long-term risk) be better used to improve conventional safety ? Further-

* Communication présentée au Congrès SFRP, "Les nouveaux enjeux de la radioprotection des travailleurs", Lyon, 11-12 juin 1991.

** COGEMA, Centre de Radioprotection dans les Mines (CRPM), Fanay, 87240 SaintSylvestre.

** CERTAC SA, BP 2, 78610 Auffargis. 
more, there appears a number ol noxious effects running against the aim pursued. As a conclusion, more efficient radiation protection could be achieved by implementation of the principle of optimization of the collective dose instead of more severe limits of individual doses.

\section{Introduction}

Peut-on encore améliorer la qualité de la protection radiologique collective dans les mines? Dans les mines d'uranium et les usines de traitement de minerai, ce problème continue de se poser malgré les récents efforts de prévention des opérateurs miniers : c'est, en effet, dans ces premières étapes du cycle du combustible nucléaire que se génère une grande partie de la dose collective et donc du risque collectif à associer à la filière nucléaire en fonctionnement normal.

En ce qui concerne le risque individuel moyen radiologique (hypothétique et à long terme) dû aux minerais d'uranium, il n'est pas, de fait, le premier des risques individuels moyens auxquels les travailleurs du sous-sol sont exposés et ceci quelles que soient les substances extraites ou le travail effectué. Mais, par rapport aux travailleurs des étapes ultérieures du cycle du combustible nucléaire, ce risque est parmi les plus élevés et les responsables de la filière souhaiteraient supprimer cette disparité dans la mesure du possible.

Le problème se pose également dans toutes les mines souterraines : le radon 222, insoupçonné jusqu'ici hors mines uraniferes, se révèle capable de produire des expositions individuelles et collectives des travailleurs radiologiquement significatives, même si la teneur en uranium des encaissants est faible. Le niveau des risques collectifs dans les mines non uranifères ne doit pas être plus élevé que celui que I'on souhaiterait dans les mines d'uranium.

Cependant, dans la mesure où les sociétés minières respectent les réglementations relatives aux limites de dose individuelle, elles ne cherchent plus, actuellement, à faire des efforts supplémentaires en vue de la réduction du risque radiologique collectif car il leur faut serrer les prix de revient. Or, il y a justement une relance du problème par le biais de l'apparition d'une perspective d'augmentation de la sévérité des limites individuelles, recommandée par la Commission internationnale de protection radiologique (CIPR) dans sa publication $n^{\circ} 60$ [4] qui devrait être introduite, à court terme dans les réglementations des Etats membres de la Communauté européenne (CE). Les sociétés minières devront revoir leur politique de protection radiologique si l'application de la publication CIPR $n^{\circ} 60$ est faite au domaine minier.

Sauront-elles faire face à des limites individuelles plus sévères et jusqu'où, non seulement techniquement mais surtout économiquement ? Quels sont les gains qui pourraient en résulter au niveau de la dose 
collective ? L'importance de ces gains sur le plan sanitaire collectif estelle suffisante pour compenser les surcoûts associés ; l'éventuelle perte de compétitivité économique des entreprises, l'éventuelle augmentation des risques autres que radioactifs ?

\section{Position du problème}

\subsection{Quelques rappels sur la doctrine de gestion des risques radiologiques élaborés par la CIPR}

Les règlements concernant la protection des populations vis-à-vis des risques d'exposition aux rayonnements ionisants font intervenir, en se plaçant dans la ligne de la doctrine de la CIPR, deux limites au niveau des doses reçues par les individus. Une première limite vue par la CIPR 60 sur des "bases raisonnables dans des opérations normales" sépare les niveaux de dose annuelle qui sont considérés comme inacceptables de ceux qui sont considérés acceptables vu la pratique industrielle reconnue comme justifiée ; la deuxième limite sépare les niveaux de dose acceptables qu'il faut gérer de ceux qui sont considérés comme non significatifs, c'est-à-dire suffisamment faibles pour que les gestionnaires des risques estiment qu'il n'y a plus exposition.

Dans le cas des expositions professionnelles subies par des travailleurs dans le_cadre de-leur-travail normal; les règlements qui suivent les recommandations proposées par la CIPR dans sa publication n²6 [2] les publications qui en dérivent, fixent ces deux limites de dose à respectivement 50 et $5 \mathrm{mSv} / \mathrm{an}$. II s'agit ici des doses effectives, avec le cumul des expositions externes et internes.

Tout industriel qui ne réussit pas à maintenir les doses individuelles annuelles de son personnel en-dessous de $50 \mathrm{mSv}$ est en infraction. Dans le domaine des niveaux de dose annuelle acceptables gérés (5 à $50 \mathrm{mSv} / \mathrm{an}$ ), l'industriel est tenu, par principe, de minimiser le risque collectif tenant compte raisonnablement des conditions économiques et techniques locales. C'est le principe de l'optimisation de la radioprotection collective présenté par la CIPR comme le principe fondamental. Pour diminuer le risque collectif, le principe de respecter des limites individuelles annuelles n'est que second et à placer dans un concept de "contraintes" où la "flexibilité" est possible (expositions "acceptables", moyenne sur 5 ans, réglementation avec limites provisoires plus élevées...).

Dans la mesure où est admise actuellement l'hypothèse de la linéarité et de l'absence de seuil dans la relation entre la probabilité d'apparition des effets sanitaires à long terme et la dose efficace, minimiser le risque collectif revient à minirniser la dose effective collective. Cette hypothèse est raisonnable dans la gamme des faibles doses effectives annuelles situées dans le domaine 5-50 mSv/an pour des expositions professionnelles pouvant se prolonger sur quelques dizaines d'années. Dans le 
domaine des niveaux de doses où la réglementation ne s'applique pas, l'industriel devra prouver en permanence qu'aucun travailleur ne dépasse la limite de $5 \mathrm{msv} / \mathrm{an}$.

\section{Remarques sur les conditions de mise en pratique aux différentes étapes du cycle du combustible nucléaire}

On constate que, vis-à-vis de ces deux limites de 50 et de $5 \mathrm{mSv} / \mathrm{an}$, les industriels du cycle du combustible nucléaire ont eu deux types de comportement. Les uns - ceux qui étaient concernés par la fabrication du combustible, son utilisation dans les réacteurs nucléaires et son retraitement, et par le stockage des déchets, c'est-à-dire toutes les opérations du milieu et de la fin du cycle - se sont donnés comme objectif opérationnel de placer tout le personnel exposé dans le domaine des niveaux de dose non significatifs, c'est-à-dire en dessous de $5 \mathrm{mSv} / \mathrm{an}$. Ils y ont réussi à quelques fractions critiques près de leurs personnels (intervention, maintenance). Ces industriels n'ont donc pas cherché à tirer parti de la tolérance offerte par le règlement pour, par exemple, réduire leur prix de revient par moins de dépenses de radioprotection. Bien que des données chiffrées sur le coût de cette option n'existent pas, il est permis de penser qu'elle correspond à un prix de l'unité de dose collective évitée très élevé, que l'intérêt sanitaire ne suffit pas à justifier. En fait, il est clair que l'objectif poursuivi par ces industriels n'est pas un objectif sanitaire mais un objectif stratégique. Ces industriels peuvent se permettre cette option parce qu'il n'y a pas encore vraiment de prix de marché pour leurs produits, ou pas de concurrence pour des services offerts sur un marché sans élasticité comme, par exemple, dans le cas du stockage des déchets radioactifs. On notera que ces industriels sont les maîtres des lieux de travail à poste fixe et des intensités des sources de rayonnements dans leurs installations. II leur est toujours techniquement possible d'intervenir sur le procédé, ou au stade de l'ingénierie de conception, pour réduire les débits de dose au niveau des postes de travail à une valeur aussi faible qu'ils l'avaient décidé, toute question de prix mise à part.

D'autres industriels, par contre, ont du mal à faire mieux que de se situer en dessous de $50 \mathrm{mSv} / \mathrm{an}$, c'est-à-dire qu'ils n'y parviennent pas toujours pour tout leur personnel. Sont ici en cause les opérations d'extraction et de traitement des minerais d'uranium, les opérations de maintenance programmées ou non des réacteurs électrogènes et des usines de fabrication des combustibles et les opérations de démantèlement et d'assainissement d'ateliers ou de sols contaminés. On notera qu'il s'agit des cas où les sources des rayonnements sont, pour les entreprises intervenantes, des sources de facto, à prendre telles qu'elles sont. Les débits de dose rencontrés dans les chantiers d'abattage miniers ou de démantèlement sont, d'ailleurs, largement inconnus au moment de la conception de ces chantiers. Mais le fait majeur est que les entreprises intervenantes sont placées en situation fortement concurrentielle. C'est le 
cas des compagnies minières qui opèrent sur un marché de l'uranium fortement déprimé depuis 10 ans. C'est le cas des sociétés de service en maintenance nucléaire ou en démantèlement radioactif qui sont nombreuses à répondre aux mêmes appels d'offre et qui doivent avoir recours à des personnels hautement qualifiés, peu abondants sur le marché du travail, et donc très recherchés. Les conditions économiques de l'obtention de la protection radiologique requise par la réglementation présente pèsent donc fortement sur la compétitivité économique de ces entreprises.

\section{Disparités des régimes réglementaires dans les différents pays producteurs d'uranium du monde à économie de marché}

On s'intéresse, ici, exclusivement aux expositions professionnelles dans les mines uranifères souterraines. Dans le tableau I, des résultats d'exposition moyenne ont été sélectionnés pour divers pays. Les sociétés qui font de la protection radiologique un objectif prioritaire ont réussi à maintenir les doses totales individuelles dans la gamme de 5 à $50 \mathrm{mSv} / \mathrm{an}$. Ce qui revient à dire que les expositions individuelles respectent l'inégalité (1), issue de la publication CIPR 26 et des publications subséquentes relatives aux mines d'uranium et introduite dans le règlement français (décret $n^{\circ} 89-502$ du 13 juillet 1989 complétant le règlement général des industries extractives) [7].

$$
\frac{\gamma}{50}+\frac{E A P_{222}}{20}+\frac{E A P_{220}}{60}+\frac{P}{1700}<1
$$

avec

$\gamma$ : dose annuelle d'exposition aux rayonnements externes gamma, en $\mathrm{mSv}$;

$E A P_{222,220}$ : énergie alpha potentielle inhalée annuelle des descendants à vie courte des isotopes 222 et $220 \mathrm{du}$ radon, en $\mathrm{mJ}$;

$P$ : activité totale des émetteurs alpha à vie longue inhalée annuelle, en Bq.

On appellera plus loin "taux annuel d'exposition totale" (TAET), l'expression ci-dessus ; (1) correspond à $T A E T<1$.

Cependant, il ne faut pas se cacher que d'autres sociétés productrices d'uranium ne respectent pas l'inégalité (1) pour l'ensemble de leur personnel. D'une manière générale, dans la plupart des mines d'uranium souterraines où l'évaluation des doses effectives individuelles repose uniquement sur une dosimétrie d'ambiance, comme cela a été le cas en France jusqu'en 1983, on doit soupçonner qu'une fraction inconnue de personnels non identifiables est en situation de dépassement de la valeur 1 du TAET, même si les résultats annoncés ne le laissent pas entendre. 
TABLEAU I

Sélection de quelques résultats d'exposition individuelle en mine d'uranium* (moyenne annuelle en mSv/an).

A selection of some data on personal exposure in uranium mines

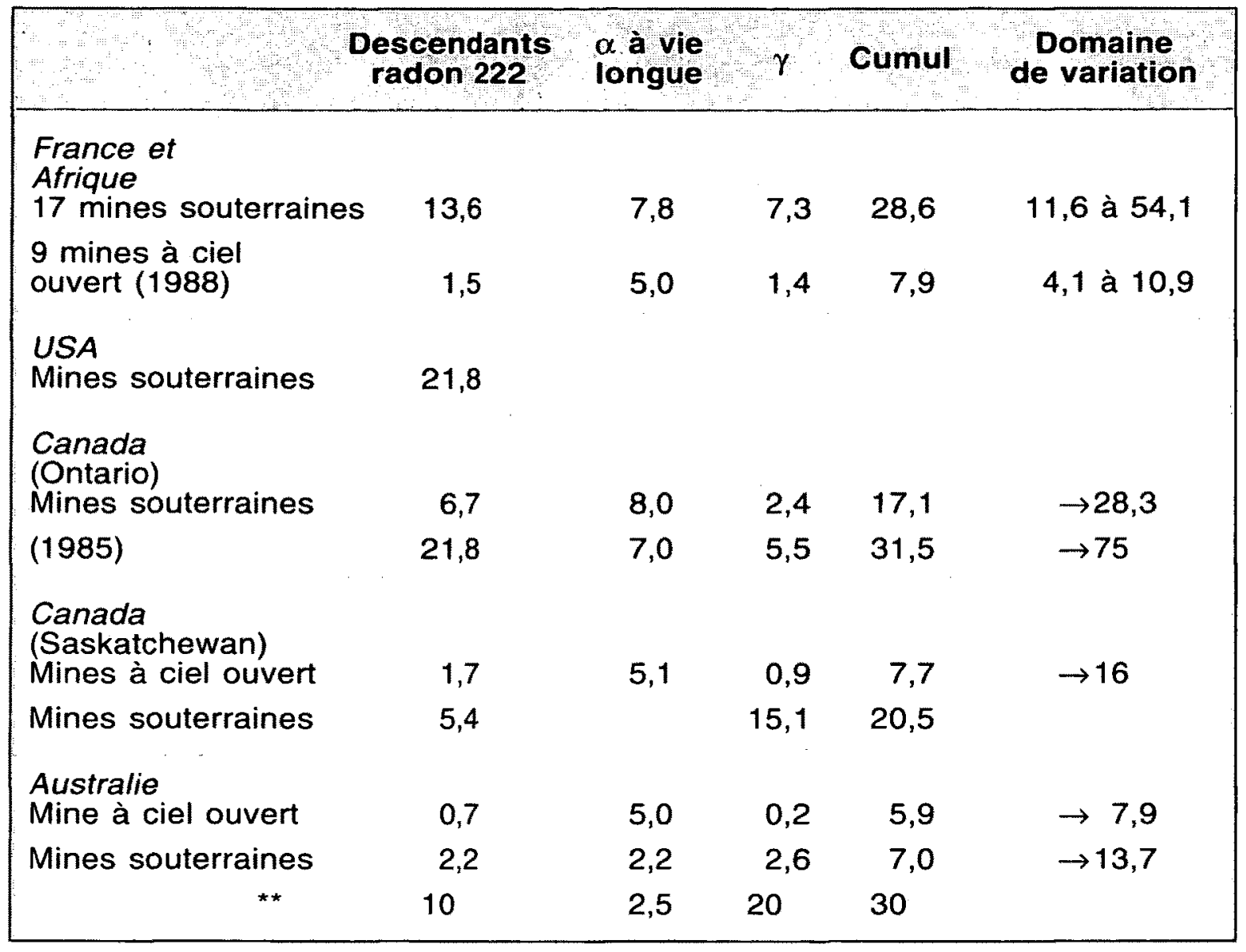

* Les données autres que françaises sont fournies par S. Frost (Canada) et R. Fry (Australie).

** Estimée en phase de conception.

En effet, en mine souterraine, vu la variabilité spatiale et temporelle dans les différents chantiers, vu la mobilité du personnel dans la mine, la distribution des doses effectives individuelles vraies présente un écart type géométrique $\sigma g$ qui se situe couramment dans la gamme 1,8< $\sigma g$ $<2,8$ (fig. 1). Or, les valeurs annoncées de la médiane $m$ de ces distributions ne sont souvent pas éloignées de $50 \mathrm{mSv}$. Pour des valeurs de $m$ se situant dans la gamme de 10 à $20 \mathrm{mSv}$, la figure 1 montre que 1 à $15 \%$ du personnel est en situation de dépassement (hypothèse de la distribution lognormale des doses).

Pour éviter ces dépassements annuels, il faut pouvoir gérer, individu par individu, mois par mois, les doses annuelles des personnes des groupes les plus exposés (ce qui revient à sortir de l'hypothèse de lognormalité). Or, en dosimétrie d'ambiance, les erreurs d'estimation sur les valeurs des doses individuelles mensuelles sont de plus de $100 \%$ sur plus de la moitié du personnel. Vouloir gérer les dépassements potentiels individu par individu, c'est-à-dire changer de poste celui-ci plutôt que celui-là, est dans ces conditions illusoire. 


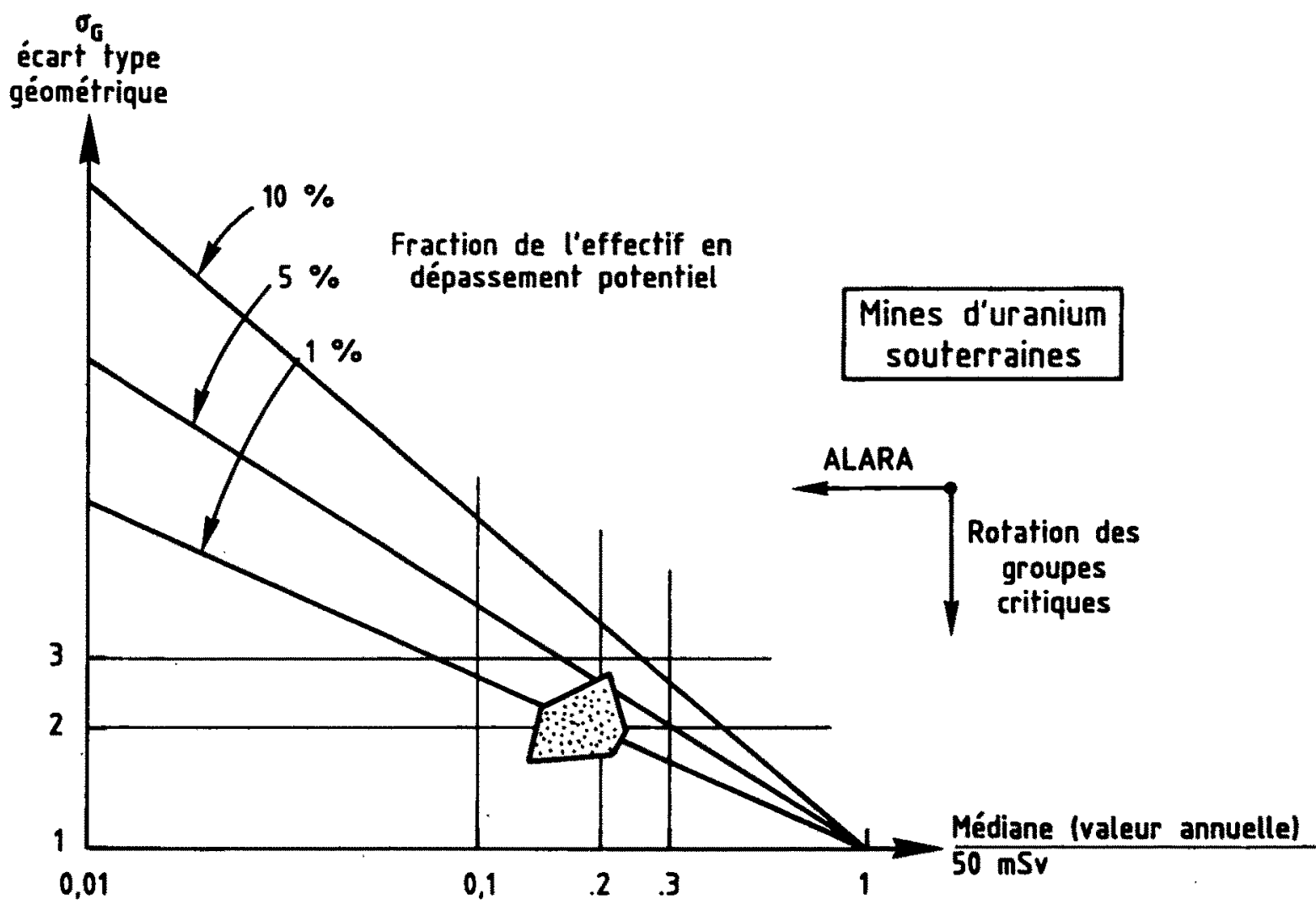

Fig. 1. - Dépassements potentiels de la limite de 50 mSv/an (hypothèse : distribution lognormale pour tout l'effectif).

Potential excesses of the $50 \mathrm{mSv} / \mathrm{y}$ limit (assumption : lognormal distribution for the whole workforce - underground uranium mines).

Mis à part ces situations ambiguës, certaines compagnies minières ont pu obtenir des autorités nationales compétentes de ne pas tenir compte de l'exposition interne par les émetteurs alpha à vie longue inhalés avec les poussières. Au Canada par exemple, la règle du cumul des équivalents de dose ne s'applique pas, les limites gamma et EAP ne sont à respecter que séparément. Or, en mine souterraine, le risque dû aux émetteurs alpha à vie longue vaut 1 à 3 fois le risque gamma et représente, selon les climats, les gisements, les méthodes minières, de 20 à $50 \%$ du risque cumulé total. Dans les mines à ciel ouvert, il peut représenter 50 à $80 \%$ du risque cumulé total. On conçoit que ne pas en tenir compte est un avantage et que ne pas cumuler en est un autre.

Dans d'autres pays, au Niger et au Gabon par exemple, les réglementations mises en place par les autorités nationales compétentes ne sont pas aussi strictes que celles qui adoptent l'inégalité (1), ceci en plein accord avec I'AIEA (Agence internationale de l'énergie atomique) qui, en connaissance de cause, a recommandé de tenir compte des particularités locales sur le plan sanitaire et sur le plan des besoins en devises. 
Toutefois, les compagnies minières de ces pays ont opté pour une dosimétrie individuelle stricte externe et interne et elles possèdent une connaissance satisfaisante des doses individuelles vraies. Les éléments sont donc en place pour faire évoluer, dans la rationalité, la distribution des doses effectives, jusqu'à ce que puisse être respectée, par exemple, lorsque ce n'est pas déjà le cas, une inégalité telle que (1).

On conçoit que les diverses possibilités d'échapper à la rigueur des recommandations issues de la publication CIPR $n^{\circ} 60$ introduisent des disparités dans la compétitivité économique des sociétés minières d'uranium. Le tableau II apporte quelques indications sur le prix de la radioprotection dans les mines d'uranium.

TABLEAU \|

Coût de la radioprotection dans les mines d'uranium (1989) pour 5 exploitations françaises et africaines.

Radiation protection costs in 5 French and African uranium mines (1989)

\begin{tabular}{|lcc|}
\hline Exploitation & FF par travailleur & FF par kg d'U extrait \\
\hline 1 (TMS $^{\star}+\mathrm{MCO}^{\star \star}$ & 8000 & 6 \\
2 (TMS) & 10800 & 4 \\
3 (TMS) & 3460 & 1,20 \\
4 (MCO) & 913 & 0,8 \\
5 (TMS) & 7200 & 2 \\
\hline
\end{tabular}

* TMS : travaux miniers souterrains.

** MCO : mine à ciel ouvert.

Remarque: Pour les TMS, les valeurs indiquées ne tiennent pas compte des coûts liés à l'aérage.

\section{Résultats à attendre d'une éventuelle augmentation de la sévérité des limites. Cas des mines}

A la suite de considérations complexes, dont on ne discute pas ici le bien fondé ni dans le cas général, ni dans leur application aux mines, la CIPR est entrée dans un processus de révision, à la sévérité, de ses normes de base et de ses limites secondaires. Les publications 60 et 61 [4-5] introduisent d'ores et déjà des modifications importantes dans l'inégalité (1) (limite d'acceptabilité), la référence gamma passant de 50 à $20 \mathrm{mSv}$, et la référence émetteurs alpha à vie longue passant de 
$1700 \mathrm{~Bq}$ à environ 800. La référence énergie alpha potentielle (EAP), issue de la publication $n^{\circ} 47$ [3] n'est pour l'instant pas modifiée, mais pourrait l'être prochainement, à la suite de deux révisions indépendantes et conduites en parallèle. La figure 2 rassemble toutes les perspectives de recommandations venues et à envisager en ce qui concerne le calcul du TAET et la définition du domaine d'acceptabilité.

\section{Formule actuelle}

CIPR N $26+47$

$$
\frac{\gamma(\mathrm{mGy})}{50}+\frac{\operatorname{EAP}(\mathrm{mJ})}{20}+\frac{\alpha(\mathrm{Bq})}{1700}<1
$$

CIPR $60+47$

$$
\frac{\gamma(m G y)}{20}+\frac{\operatorname{EAP}(m J)}{20}+\frac{\alpha(B q)}{830^{*}}<1
$$

CIPR $60+$ nouveau modèle pulmonaire

$$
\frac{\gamma(\mathrm{mGy})}{20}+\frac{\operatorname{EAP}(\mathrm{mJ})}{7,5}+\frac{\alpha(\mathrm{Bq})}{300}<1
$$

CIPR $60+$ nouvelle approche des enquêtes épidémiologiques

$$
\frac{\gamma(\mathrm{mGy})}{20}+\frac{\operatorname{EAP}(\mathrm{mJ})}{20 \times Y}+\frac{\alpha(\mathrm{Bq})}{830}<1
$$

avec $Y>$ ou $<1$

Fig. 2. - Formules envisageables pour le calcul de TAET.

Possible formulas for TAET calculations.

* Calcul fait à partir des nouvelles LAI (CIPR 61) des classes les plus sévères en inhalation des 5 émetteurs alpha concernés. 
Actuellement, la valeur de la dose reçue à partir de laquelle le classement des travailleurs est imposé est égale à la limite à ne pas dépasser pour les personnes du public $(5 \mathrm{mSv} / \mathrm{an})$. Or, la CIPR propose de passer de 5 à $1 \mathrm{mSv} / \mathrm{an}$. Si l'on applique $1 \mathrm{mSv}$ au cas du risque EAP, et en gardant le facteur de risque de la publication CIPR 47 [3] cela reviendrait à classer toute personne travaillant dans une ambiance où l'activité volumique en radon 222 est égale à environ $100 \mathrm{~Bq} \cdot \mathrm{m}^{-3}$ en moyenne sur le temps de travail (hypothèse $F=0,3$ ). Ce n'est pas beaucoup, il suffit d'être en milieu souterrain ou dans certains locaux.

Quels résultats sont à attendre de ces futures limites éventuelles ?

Les sociétés ne respectant pas les recommandations de la CIPR vont continuer à le faire. Parmi celles qui respectent actuellement les recommandations de la CIPR selon l'inégalité (1), beaucoup, avec l'accord tacite ou explicite de leurs autorités nationales compétentes, vont rejoindre les précédentes, car respecter ces recommandations mettraient en jeu leur viabilité économique. En ce qui concerne les sociétés qui prendront les dispositions nécessaires pour s'adapter à des limites individuelles plus sévères, on n'observera pas nécessairement une amélioration de la qualité de la protection radiologique collective, ce qui, pourtant, devrait être l'objectif principal. La mise en œuvre de ces limites dans le cas des mines peut, en effet, avoir des effets pervers, ci-après analysés.

\subsection{Effets pervers potentiels de l'abaissement de la limite d'acceptabilité}

Considérons les Etats qui appliqueraient immédiatement et sans "flexibilité" l'un des modes de calcul potentiels plus ou moins sévères exposés en figure 2. Les améliorations qui seraient obtenues sur le plan des risques individuels des travailleurs les plus exposés ("groupes critiques") pourraient réduire le risque collectif seulement si les sociétés y parviennent par la réduction des débits de dose aux postes de travail ou par l'augmentation de la productivité de l'extraction du minerai. Or, l'accent étant mis sur la limite individuelle d'acceptabilité, certaines sociétés minières vont être tentées d'obtenir le respect strict de la réglementation par la rotation du personnel. II s'agit, pour un même nombre d'heures de travail en mine, d'utiliser un plus grand nombre de personnes. La CIPR ouvre la porte à cette solution échappatoire, en admettant que la limite de dose effective de $20 \mathrm{mSv} / a n$ pourrait n'être appliquée qu'en moyenne sur 5 années consécutives, la dose effective annuelle étant de toute facon lirnitée à $50 \mathrm{mSv} / \mathrm{an}$. Une société, si sa structure et l'environnement industriel le lui permettent, peut théoriquement affecter 2 ans de suite son personnel au travail de la mine, puis sur les 3 ans qui suivent l'affecter à des travaux n'impliquant pas de suivi dosimétrique ou procéder à son renvoi. Or, l'effet pervers est qu'outre le non-respect de la CIPR dans son esprit, la rotation implique, en fait, une plus grande dose collective et un niveau de risque conventionnel minier plus grand. On pousse ainsi les sociétés qui pourraient appliquer la rotation du personnel à aller à l'encontre des vrais objectifs de protection de ce personnel. 
Envisageons, maintenant, les sociétés minières qui voudront jouer honnêtement le rôle de sociétés respectueuses de la protection édictée par les règlements de leur pays. Les sociétés d'extraction de minerais d'uranium existantes pourront, dans un premier temps, en faisant faire l'expertise appropriée des conditions de la formation de leur dose collective, trouver des morceaux de cette dose collective à faire disparaître à coût faible. Cependant, au fur et à mesure que des progrès seront faits, les coûts seront de plus en plus élevés. Si, de plus, dans le calcul du TAET, l'importance du terme EAP devait être augmentée, seul un nombre limité de sociétés pourront maintenir leur exploitation. Encore, ce résultat sera-t-il obtenu pour un prix de l'unité de dose collective évitée supérieur à la valeur monétaire du gain sanitaire théoriquement obtenu par cette dose évitée, c'est-à-dire que l'on aura dépassé le niveau de protection radiologique optimal au sens de la CIPR. L'effet pervers est le conflit qui apparaît entre le principe de l'optimisation et le principe de limitation des doses individuelles. Ici, il faut remarquer qu'au niveau des risques actuels, le risque radiologique n'est qu'un des risques, et de loin pas le premier, auxquels s'exposent professionnellement les mineurs. On en conclut tout de suite que les sommes qui seraient dépensées par la société minière dans la réduction du risque radiologique, somme toute hypothétique et à long terme, seraient plus efficacement utilisées pour l'amélioration des autres postes de la sécurité conventionnelle.

Considérons, maintenant, les sociétés existantes qui, pour des rai= sons techniques ou économiques, ne pourront pas se mettre en conformité avec la nouvelle réglementation. Elles devraient s'arrêter. Or, dans certains pays, les désavantages économiques et sociaux de ces fermetures seront très supérieurs aux détriments sanitaires hypothétiques qui auront pu être évités.

Quant aux sociétés qui souhaiteraient ouvrir une nouvelle mine d'uranium, elles hésiteront car la marge de rentabilité aura dirninué. De plus, elles craindront que les groupes s'opposant traditionnellement à l'industrie nucléaire, comme au Canada ou en Australie, ne se trouvent confortés par la sévérité supplémentaire d'une nouvelle réglementation, ce qui signifierait, en tout état de cause, des délais et des coûts supplémentaires avant l'ouverture de la mine et le moment de l'arrivée des premiers retours financiers. Beaucoup de nouveaux projets seront mis en attente dans tous les pays. La prospection minière de l'uranium ne progressera plus. L'extraction de l'uranium sera une aventure trop risquée pour un investisseur, même s'il s'agit des gisements riches du Canada. Or, depuis 1985, la production d'uranium est inférieure à la consommation des stocks (fig. 3, d'après [8]). Ceux-ci (100 000 tonnes dans le monde à économie de marché), au rythme actuel, devraient s'épuiser assez vite. Certains experts estiment que l'on risque de se trouver, d'ici à 10 ans, en situation de rupture des approvisionnements et que, vu les délais d'ouverture de nouvelles mines, il y a donc instauration d'une situation qui tendrait à freiner le remplacement du charbon par le nucléaire. Or, les inconvénients écologiques et sanitaires, et peut-être surtout climatiques, 


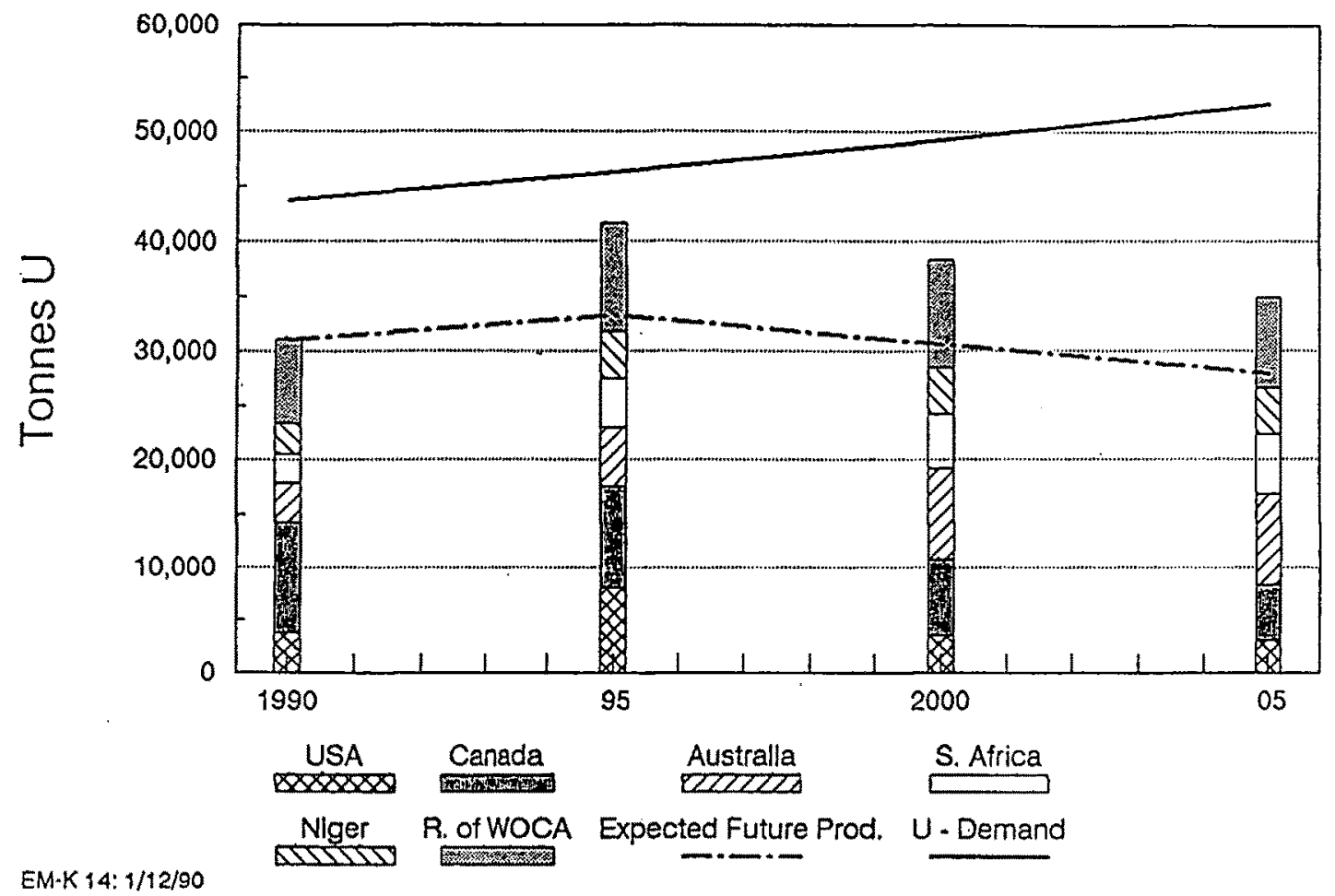

Fig. 3. - Projection MEM de la demande et de la production d'uranium, d'après [8]. WOCA uranium supply-demand projections, after [8].

du charbon sont probablement aussi importants. Un autre effet pervers à moyen et long terme est donc qu'il y a conflit entre les intérêts étroitement liés à la protection radiologique des groupes critiques de la population des mineurs d'uranium et des intérêts économiques et environnementaux d'une bien plus grande ampleur.

Considérons maintenant les rnines de substances non radioactives. En France, elles sont prises en compte pour la radioprotection dans la réglementation générale minière (RGIE), au même titre que les mines d'uranium [7]. Cependant, il est en général possible, pour toutes ces mines, de se maintenir en dessous du 1/3 de la limite annuelle d'acceptabilité (moins de $15 \mathrm{mSv} / \mathrm{an}$ pour à peu près tout leur personnel). Les sociétés sont donc autorisées, réglementairement, à n'évaluer les doses individuelles que par la procédure de la dosimétrie de fonction (dosimètres individuels) pour seulement 10 à $20 \%$ du personnel. Des résultats sur les niveaux d'exposition sont donnés dans le tableau III. Si $20 \mathrm{mSv} / a n$ devient la limite d'acceptabilité, $15 \mathrm{mSv}$ devient alors supérieur au 1/3 de la limite et tous les individus devront être équipés de dosimètres individuels. II s'ensuivra un surcoût non négligeable pour un exploitant de l'ordre de $3500 \mathrm{FF} / a n$ par travailleur. Cette amélioration de la comptabilité dosimétrique n'apporterait pratiquement aucune réduction 
TABLEAU III

Résultats d'expositions individuelles dans les mines non uranifères. Personal exposures in non-uranium mines

1) Données recueillies dans 60 mines d'Europe*

\begin{tabular}{|ccc|}
\hline Ambiance moyenne sur le retour dlair général & $\begin{array}{c}\text { Gamme dexposition } \\
\text { moyenne calculée } \\
\text { (mSv.an-1) }\end{array}$ \\
\hline 14 & Radon $222\left(\mathrm{~Bq} \cdot \mathrm{m}^{-3}\right)$ & $<1$ \\
24 & 100 à 500 & 1 à 5 \\
7 & 500 à 1000 & 5 à 10 \\
4 & 1000 à 2000 & 10 à 20 \\
11 & $<2000$ & $<20$ \\
\hline Total 60 & & \\
\hline
\end{tabular}

* d'après S. Bernhard, J. Le Gac, H. Seguin, P. Zettwoog. "Radon levels and radon daughters exposures of workers in non uranium mines of the E.C." In : International conference on radiation hazard in mining, Golden, October 4-9, 1981. Golden : Colorado School of Mines, 625-628.

2) Données recueillies au Royaume-Uni**

\begin{tabular}{|c|c|c|c|c|}
\hline \multicolumn{5}{|c|}{$\begin{array}{ll}\text { Répartition des doses annuelles (mSv) } \\
\end{array}$} \\
\hline \multicolumn{5}{|c|}{ Minéral Nombre de travailleurs } \\
\hline 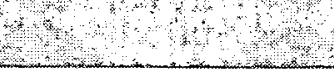 & $0-5$ & 5-15 & 15-50 & $>50$ \\
\hline Barite & 10 & - & - & - \\
\hline Argile & 22 & 12 & - & - \\
\hline Spathfluor & 75 & 10 & 55 & - \\
\hline Gypse & 181 & - & 57 & - \\
\hline Calcaire & 7 & 21 & 11 & - \\
\hline Métaux ferreux & 2 & 45 & 110 & - \\
\hline Grès & 7 & - & - & 3 \\
\hline Ardoises & 57 & - & - & - \\
\hline Autres & 668 & - & 4 & - \\
\hline Charbon & 801 & 59 & 10 & \\
\hline
\end{tabular}

* d'après D.A. Bottom, O.W. Dixon, D. Gooding. "Exposure to radon in British mines". Congrès BNES, 1991. 
de la dose collective. Elle serait suffisamrnent coûteuse pour que certaines entreprises, à la limite de la rentabilité économique, doivent fermer. De nouveau, il y a un effet pervers, un détriment économique et social qui naît sans même apporter un avantage sanitaire en contrepartie.

\subsection{Effets pervers potentiels de l'abaissement de la limite en dessous de laquelle les travailleurs ne sont pas considérés comme exposés}

Cette limite sera-t-elle, comme actuellement, égale à 1/10 de la limite d'acceptabilité, soit $2 \mathrm{mSv} / \mathrm{an}$, ou égale à la limite proposée pour le public soit $1 \mathrm{mSv} / \mathrm{an}$ en moyerme sur 5 ans ? Avec l'une ou l'autre de ces valeurs, si elles devaient être adoptées, les conséquences économiques locales seraient plus irnportantes que celles de la lirnite d'acceptabilité.

Sont concernées, en effet, toutes les entreprises qui travaillent en milieu souterrain et qui pouvaient, jusqu'ici, sans aucun effort particulier pour contrôler le risque EAP radon 222 maintenir leur personnel en dessous de la limite de $5 \mathrm{mSv} / \mathrm{an}$. Elles ne pourront pas, par contre, pour la plupart, respecter la limite de 1 ou $2 \mathrm{mSv} / \mathrm{an}$ car le coût des assainissements nécessaires serait trop élevé. II s'agit, en effet, de niveaux d'exposition en $E A P$ radon 222 qui sont du domaine des niveaux de l'exposition naturelle et qui sont relativement incompressibles, pour peu que la géologie soit défavorable.

Soulignons qu'il ne s'agit plus seulement des mines mais aussi des entreprises de creusement de tunnels ou de réservoirs souterrains, des champignonnières, du métro et des parkings, et de toute entreprise qui offre du travail en sous-sol. Toutes ces entreprises devront se livrer à une investigation dosimétrique et on constatera que leurs travailleurs seront considérés cornme des travailleurs exposés à I'EAP des descendants du radon 222 . Elles devront donc procéder en routine à la surveillance radiologique de leur personnel. Elles verront leurs charges augmentées de coûts bureaucratiques, dosimétriques et médicaux qui ne produiront strictement aucun avantage sanitaire, ni individuel, ni collectif. L'effet pervers est donc la diminution de la compétitivité des entreprises sans contre-partie pour la collectivité.

L'ironie est que la plupart de ces travailleurs seront exposés chez eux à des niveaux EAP radon 222 conduisant à des doses effectives supérieures à 1 ou $2 \mathrm{mSv} / \mathrm{an}$, certains d'entre eux pouvant y recevoir des doses effectives à la limite des $20 \mathrm{mSv} / \mathrm{an}$. La CIPR recommande de n'intervenir pour assainir les habitations à risque EAP radon 222 élevé que pour des doses effectives des habitants supérieures à $20 \mathrm{mSv} / \mathrm{an}$. L'effet pervers est ici une absence de logique qui sera évidente aux employeurs comme aux travailleurs.

On ne peut pas, sans créer une situation inacceptable de double standard entre les entreprises d'un même Etat, accepter que les mines et usines d'exploitation des minerais d'uraniurn, de terres rares ou de phosphates, par exemple, soient obligées de respecter des limites beaucoup 
plus sévères que si elles étaient des entreprises ne travaillant pas dans le nucléaire. Si la valeur de $5 \mathrm{mSv} / a n$ était maintenue pour tous, alors la limite d'acceptabilité de $20 \mathrm{mSv} / \mathrm{an}$ en serait beaucoup trop proche, un facteur 4 en dose ne suffit pas pour qu'on puisse prétendre que l'on passe d'un effet biologique non significatif à un effet biologique inacceptable. L'effet pervers, ici, est que l'ensemble de l'édifice est mis en cause.

\section{Perspectives d'une amélioration de la qualité de la protection radiologique dans les mines d'uranium}

En introduction, l'attention a été attirée sur la nécessité d'obtenir une amélioration de la qualité de la protection radiologique collective dans les mines d'uranium ; c'est à cette étape du cycle du combustible nucléaire que le coût radiologique spécifique est le plus élevé.

On a exposé, au chapitre 4, que l'augmentation de la sévérité des limites individuelles actuelles n'apportaient pas forcément une amélioration de la protection radiologique collective. De plus, elle induit toute une série d'effets pervers qui ruinent la crédibilité du dispositif de protection.

On montre, maintenant, que des perspectives d'amélioration de la protection radiologique collective que l'on peut envisager cette fois-ci dans le cadre de la mise en œuvre du principe d'optimisation de la CIPR sont,..jusqu'à un certain niveau, tout à fait réelles. Notons, d'ailleurs, que, dans la mesure où les écarts-types géométriques des distributions des doses individuelles sont maîtrisés, à la réduction de la dose collective correspond aussi, de surcroît, la disparition progressive des groupes critiques les plus exposés. C'est pourquoi, dans la suite, on ne s'intéresse plus à la suppression des doses individuelles en dépassement mais seulement à la réduction de la dose effective collective, sous contrainte technique et économique. Elle entraîne automatiquement la réduction du risque individuel moyen.

De façon à pouvoir comparer les performances de différentes mines en ce qui concerne la protection radiologique collective, nous introduisons l'irradiation spécifique, rapport de la dose collective annuelle (homme-sievert par an) à la quantité d'uranium contenu dans les minerais extraits par an. De même, nous introduisons le coût spécifique de la radioprotection, rapport des dépenses dans le domaine à la même quantité d'uranium. Les valeurs relatives des 3 composantes de la dose collective effective totale dans les mines d'uranium souterraines et à ciel ouvert sont mises en évidence dans la figure 4. A titre d'exemple, des valeurs de l'irradiation spécifique d'un certain nombre de mines de France et d'Afrique sont préséntées (fig. 5). Tous les résultats dosimétriques utilisés ici sont obtenus à l'aide du même appareil de mesure, à savoir le dosimètre individuel commercialisé par le CRPM*. Ils sont donc

* CRPM : Centre de radioprotection dans les mines. 
bien directement comparables. On prendra note de la variabilité des valeurs de l'irradiation spécifique dont on a pu très bien rendre compte par une modélisation qui ne fait intervenir que les paramètres techniques de l'exploitation [10].

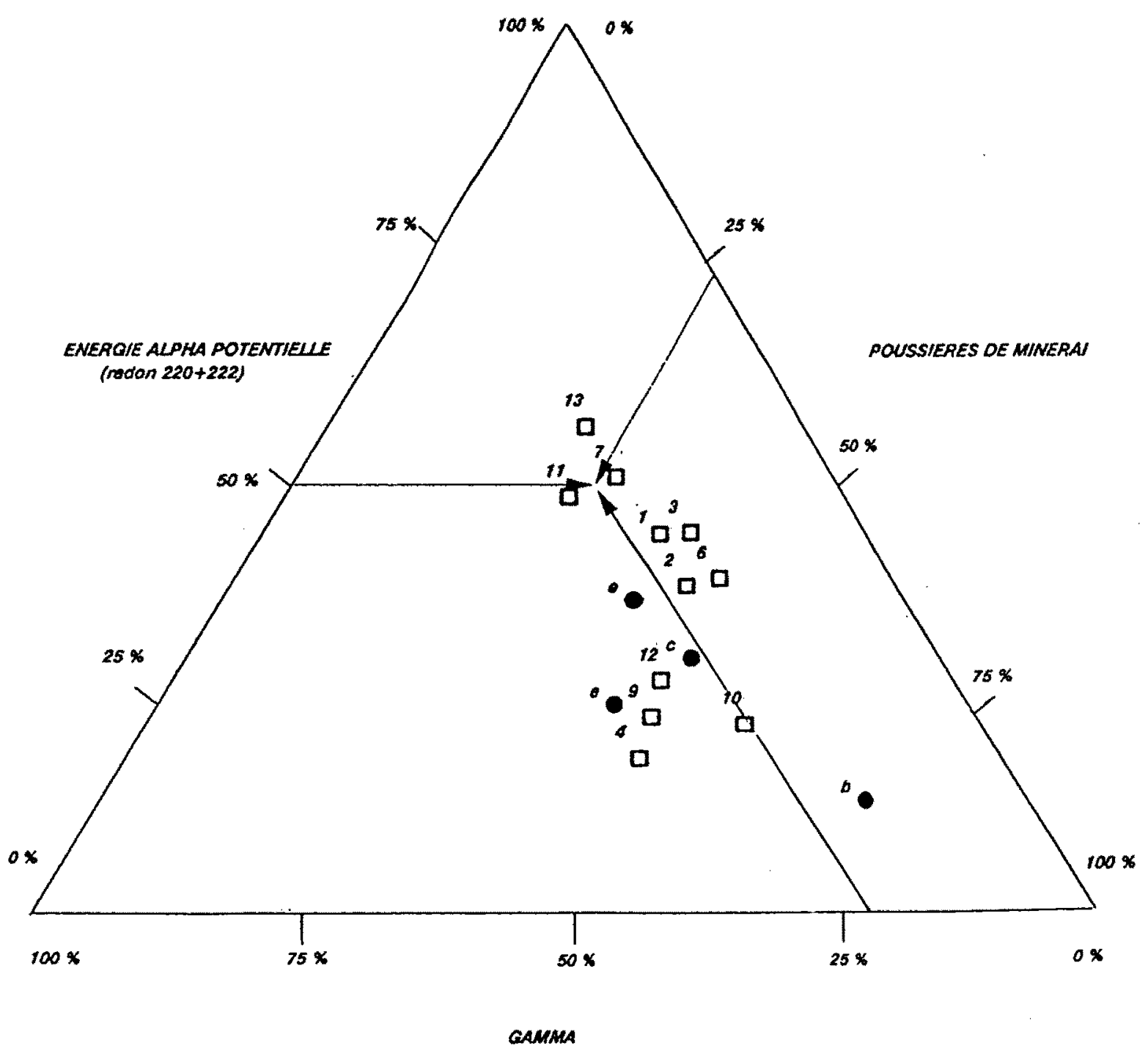

Fig. 4. - Importance de chaque risque dans le cumul des expositions pour les exploitations françaises en 1990.

Contribution of the three components of the risk to the total dose equivalent for French uranium mines in 1990.

- Travaux miniers souterrains

- Mines à ciel ouvert. 


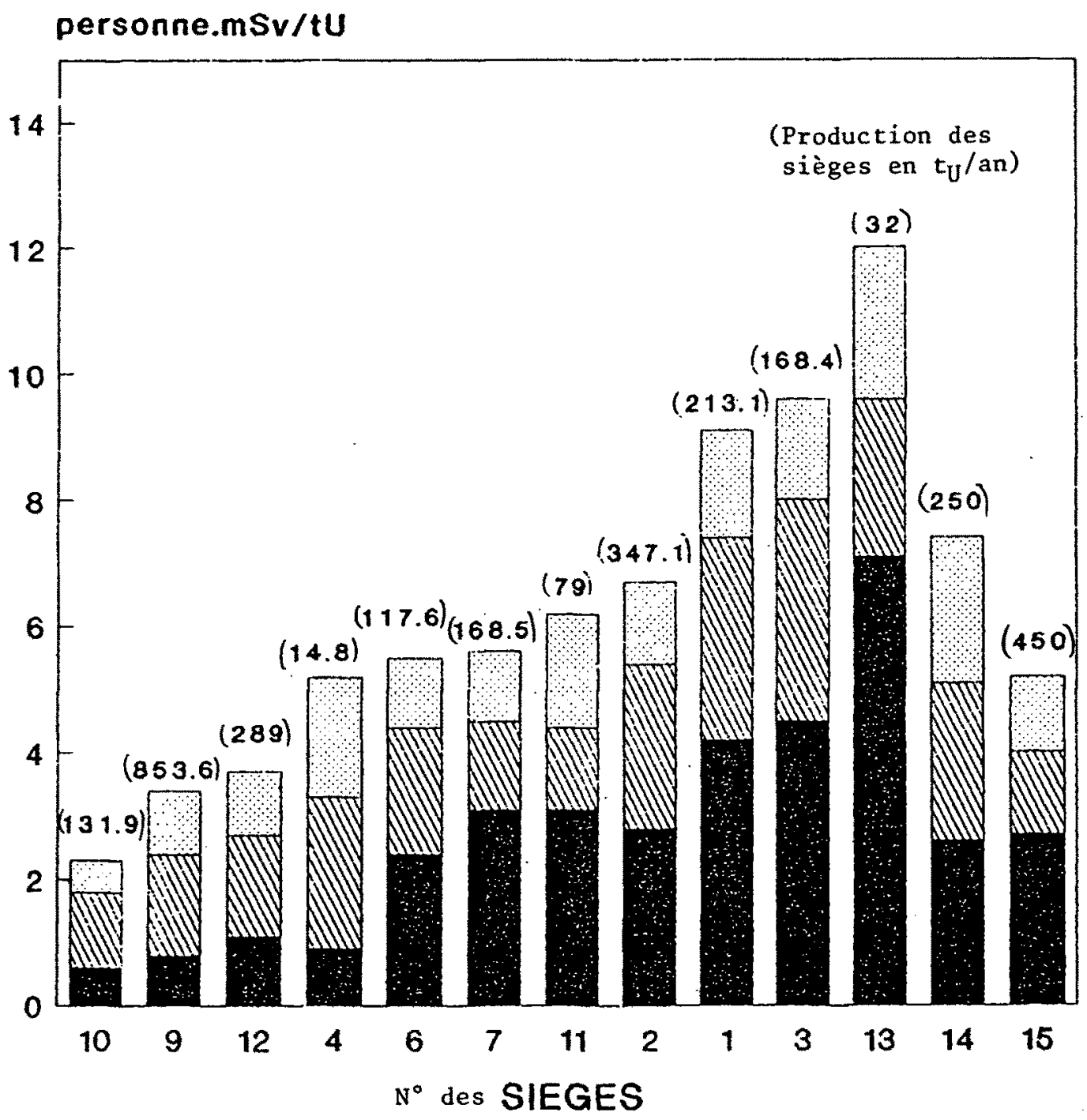

EAP $220+222$ M GIII POUS. WMA

Fig. 5. - Irradiations spécifiques totales - travaux miniers souterrains, année 1990, France et Gabon.

Collective specific exposures, underground mines, France and Gabon, 1990.

\subsection{Variabilité des facteurs explicatifs de la dose collective}

La dose effective collective s'explique par des facteurs d'ordre géologique, des facteurs opérationels liés à la méthode d'exploitation et par des facteurs organisationnels. Or, au fur et à mesure que l'exploitation progresse, tous ces facteurs varient temporellement et spatialement de façon importante et de manière aléatoire (en ce qui concerne les facteurs géologiques). C'est pourquoi l'optimisation ne peut être obtenue une fois pour toutes. C'est le résultat d'une démarche itérative qui ne s'arrête qu'avec le démantèlement de la mine. 


\subsection{Conséquences de cette variabilité}

L'exploitant qui veut maîtriser la formation de sa dose collective doit mettre en place un dispositif de collecte de données l'avertissant, en temps réel, des évolutions défavorables des sources de rayonnements et des performances des matériels d'assainissement des infrastructures et des postes de travail, et, par ailleurs, avoir constarnrnent à sa disposition les moyens techniques permettant de rétablir les situations sans perte de temps dès que des anomalies sont detectées. La collecte et le traitement des données de routine sur la formation de la dose collective et les remèdes doivent être organisés en fonction des différents temps de réaction. C'est ce que nous avons appelé l'approche dynamique de l'optimisation [6].

\subsection{Mise en ceuvre de l'optimisation}

L'exploitant qui s'est équipé matériellement et intellectuellement dans la perspective de la mise en œuvre d'une optimisation dynamique ne manquera pas de réaliser en quelques années des progrès substantiels au niveau de ses irradiations spécifiques.

\subsubsection{La phase préliminaire de la suppression des gaspillages}

Notre expérience est que, dans une mine qui n'a pas été expertisée sur le plan de la radioprotection, on observe que l'on peut, dans un premier temps, à la fois supprimer certaines fractions de la dose collective et réaliser des éconornies sur le coût de la protection. Après le départ des experts, pour que la situation ne se dégrade pas à nouveau, une action de sensibilisation et de formation auprès du personnel est à organiser. Sur la figure 6 , la réduction à coût négatif ou nul de la dose collective sur le trajet $A_{0} A_{3}$ est obtenue par des actions qui, en fin de compte, ne relèvent que du bon sens. Elle est schématiquement représentée en 3 troncons $A_{0} A_{1}, A_{1} A_{2}, A_{2} A_{3}$, correspondant respectivement aux trois types d'évolution à court terme, moyen terme, et long terme. Comme exemple de ce type d'action, on peut citer celui de la maintenance des dispositifs d'aérage auxiliaires des mines souterraines.

Le tableau IV montre qu'il y a encore des progrès à faire dans une mine en ce qui concerne le transport de l'air auxiliaire par des colonnes souples. Trop de résistance aéraulique, c'est-à-dire souvent trop de coudes, c'est un ventilateur qui gaspille de l'électricité en pertes de frottement pariétal, trop de fuites, c'est-à-dire trop de trous ou de mauvais raccordements, c'est une autre manière de ne pas atteindre ses objectifs tout en dépensant de l'énergie électrique nécessaire.

\subsubsection{Phase d'optimisation}

Lorsque tous les gaspillages ont été décelés et éliminés dans le cas des mines existantes, ou directement dans le cas des projets, l'exploitant doit passer à la phase de recherche de la protection optimale. Chaque option nouvelle de protection représente maintenant l'acceptabilité d'une 
augmentation du coût spécifique de protection, avec, en contrepartie, une réduction potentielle de l'irradiation spécifique. Sur la figure 6, l'ensemble des performances de chaque option se trouve dans une zone hachurée dont on a marqué en trait gras la frontière où se situent toutes les solutions optimales possibles de la politique de radioprotection. Chacun des points de cette frontière est un optimum qui correspond à un prix donné de l'homme-sievert évité. On a construit graphiquement la

\section{homme • Svtt d'uranium}

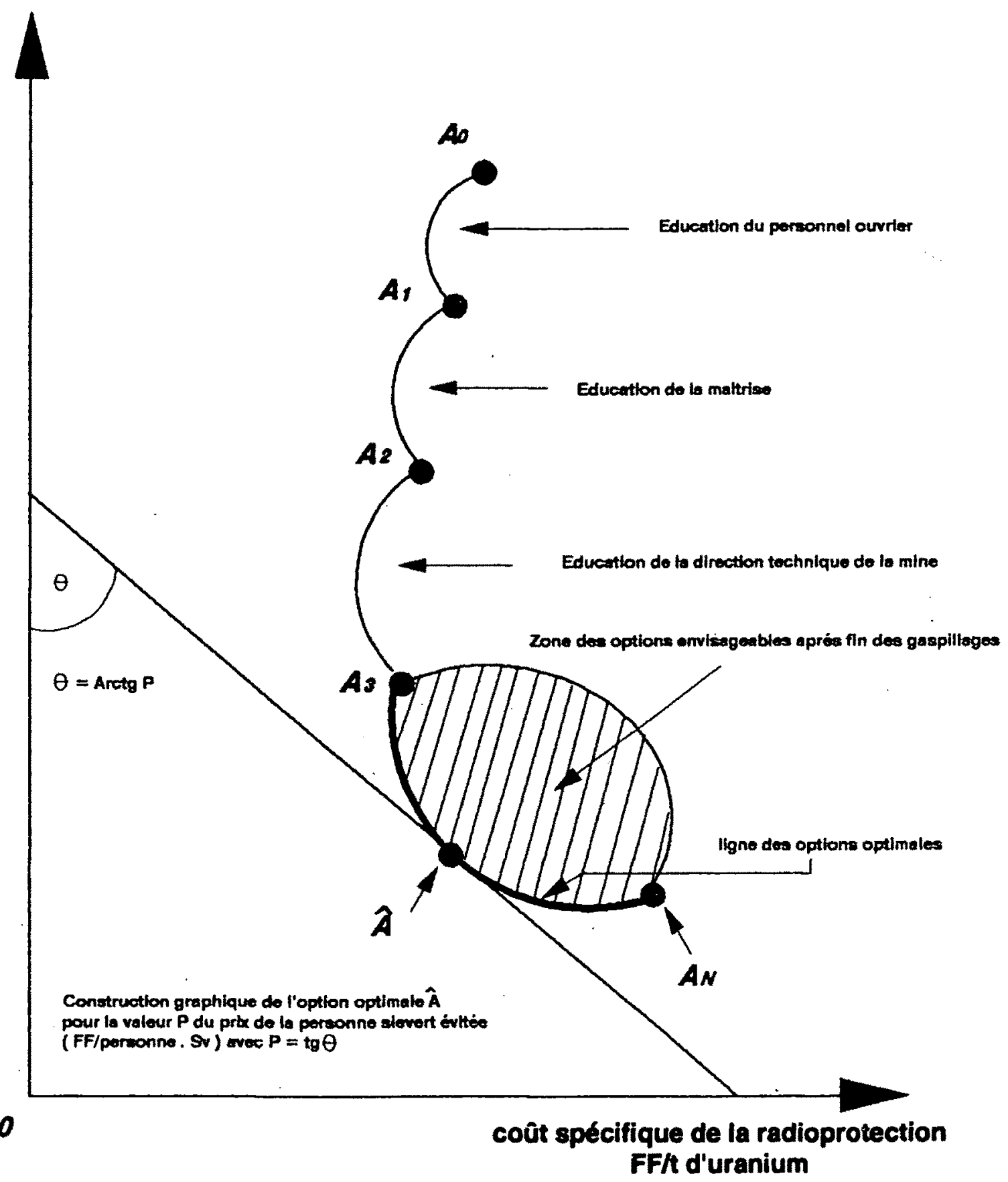

Fig. 6. - Optimisation dynamique.

Dynamic optimisation. 
TABLEAU IV

Résistance hydraulique et fuite des colonnes d'aérage : répartition des valeurs mesurées en 1990 dans les mines françaises, en pourcentage des installations contrôlées.

Hydraulic strength and ventilation column losses : distribution of French mine values measured in 1990 as a percentage of controlled installations

\begin{tabular}{|c|c|c|c|c|c|}
\hline \multirow{2}{*}{$\begin{array}{c}\text { Nombre } \\
\text { d'installations } \\
\text { contrôlées } \\
\text { en } 1990\end{array}$} & \multicolumn{5}{|c|}{ Coefficients de fuites (en $\mathrm{m}^{3} / \mathrm{s} / 100 \mathrm{~m} 1 \mathrm{kPa}$ ) } \\
\hline & $<0,2$ & 0,2 à 0,5 & 0,5 à 1 & 1 à 1,5 & $>1,5$ \\
\hline 151 & 3 & 11 & 28 & 13 & 44 \\
\hline 37 & & 5 & 30 & 11 & 54 \\
\hline 25 & & & & & 100 \\
\hline 8 & & & & & 100 \\
\hline 899 & 6 & 12 & 20 & 15 & 47 \\
\hline
\end{tabular}

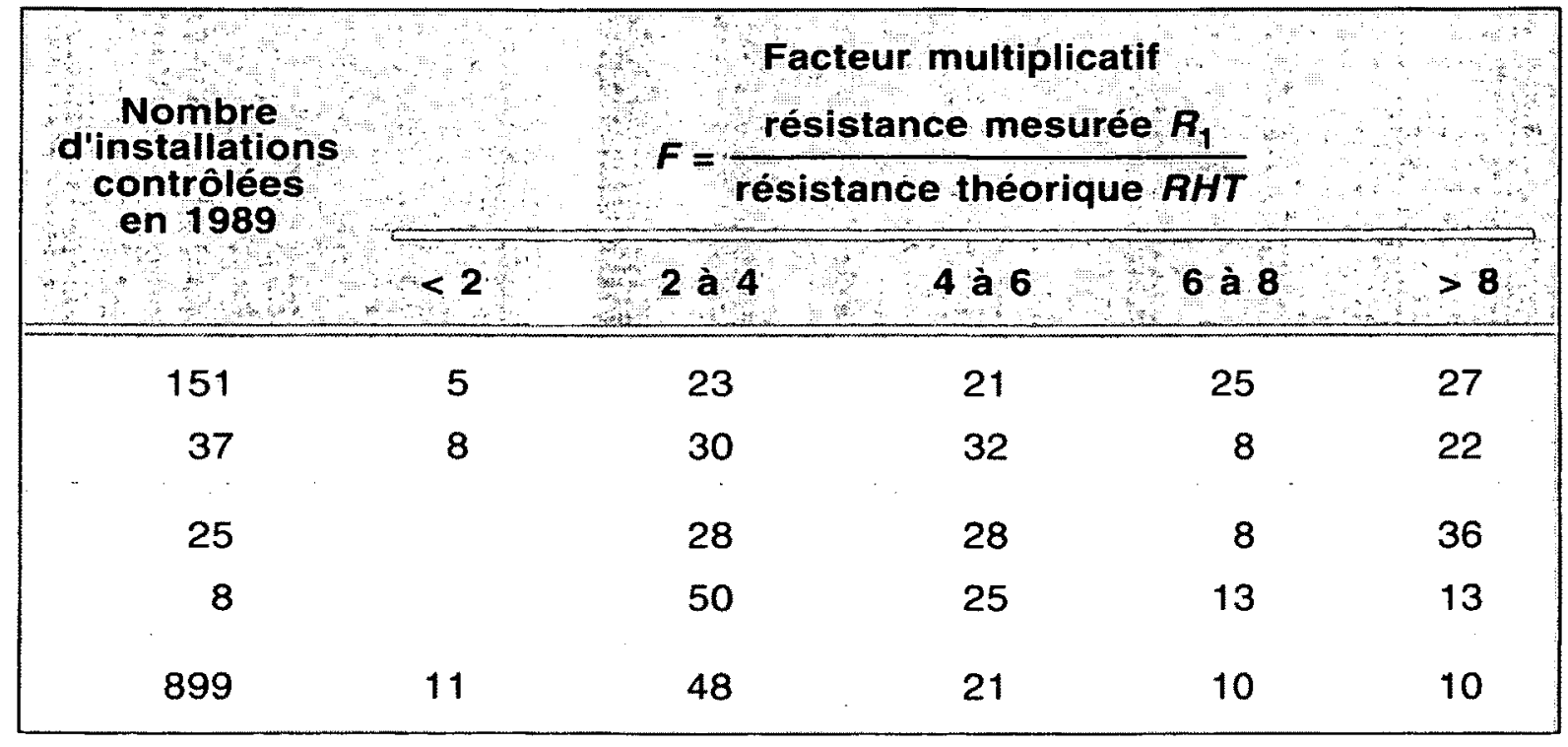

position du point $\hat{A}$ qui correspond, sur cette frontière, à l'optimum pour le prix $P$. Le prix $P$ choisi est celui qui est considéré comme raisonnable dans le pays considéré et qui tient compte de la spécificité de l'exploitation et de son environnement économique et social.

Dans le cas d'une mine existante, les paramètres phénoménologiques qui sont propres à chaque mine peuvent être ajustés de façon à rendre compte, le mieux possible, à la fois des valeurs de l'irradiation spécifique fournies par la dosimétrie et des résultats du traitement des données de terrain collectées en routine sur la formation de la dose collective. 
Dans le cas de chantiers en projet, la protection optimale est recherchée par modélisation des mécanismes de l'exposition des travailleurs [10], dont les formules semi-empiriques doivent être validées sur le terrain. On peut alors calculer le coût marginal de la réduction d'une unité de dose collective basée sur la modification de paramètres techniques sur lesquels l'exploitant peut agir. Quand ce coût atteint la valeur de $P$ acceptée, la situation optimale est obtenue et il convient de ne pas aller plus loin.

\subsubsection{La productivité d'extraction du minerai, facteur primaire du niveau des irra- diations spécifiques}

D'une manière générale, la modélisation que nous avons introduite fait intervenir comme premier facteur explicatif de l'irradiation spécifique Ir la productivité d'extraction du minerai Pdt. L'irradiation spécifique, toutes choses égales par ailleurs, lui est inversement proportionnelle. Cette loi est assez bien vérifiée pour un certain nombre de mines que nous avons eu à connaître. Par contre, on peut aussi constater une proportionnalité de Ir à l'inverse de la racine carrée de Pdt correspondant à l'intervention simultanée d'autres facteurs explicatifs.

L'exploitant a donc intérêt à introduire des méthodes minières performantes sur le plan technique. De ce point de vue, les exploitations de minerais riches, qui peuvent bénéficier de tous les progrès techniques, sont mieux placées que les exploitations anciennes, et de surcroît de minerais pauvres, où les progrès en matière de productivité d'extraction sont évidemment limités, les sections de galerie, les infrastructures, par exemple, étant pratiquement non évolutives ou exigeant des délais de mise en œuvre s'étalant sur plusieurs années. Dans les mines futures et avec des méthodes minières bien choisies, on peut espérer des performances radiologiques technico-économiques très supérieures à celles observées jusqu'ici.

\subsection{Un exemple de dérivation des coûts marginaux de réduction de la dose collective}

Le tableau $V$ donne, pour 12 mines souterraines, des valeurs de coût spécifique de la ventilation $C s$, calculées à partir d'un prix du $\mathrm{kWh}$ de $0,35 \mathrm{FF}$, et d'une ventilation primaire assurée 6000 heures par an. II donne aussi Ir (EAP) pour l'année 1989. On en a déduit le coût marginal $P$ que ces options auraient eu si la direction de la mine, en fin d'année, avait choisi de réduire sa dose collective EAP par un accroissement de l'air primaire. On a adopté, dans le tableau $V$ la valeur optimiste $K=1,5$ qui correspond à un facteur d'équilibre de l'air primaire très faible. Le tableau VI montre l'évolution du coût marginal $P$ de cette option pour 9 de ces différentes mines entre 1984 et 1989 avec, en regard, l'évolution de la dose collective. On observe que se vérifie la loi des rendements décroissants des efforts de réduction de la dose collective au fur et à mesure que celle-ci diminue. 


\section{TABLEAU $V$}

Coût marginal pour éviter $1 \mathrm{~h} \cdot \mathrm{Sv}$ dans le cadre de l'option "accroissement du débit d'aérage primaire". Marginal cost $(P)$ of collective dose reduction if the primary ventilation rate is increased (1989)

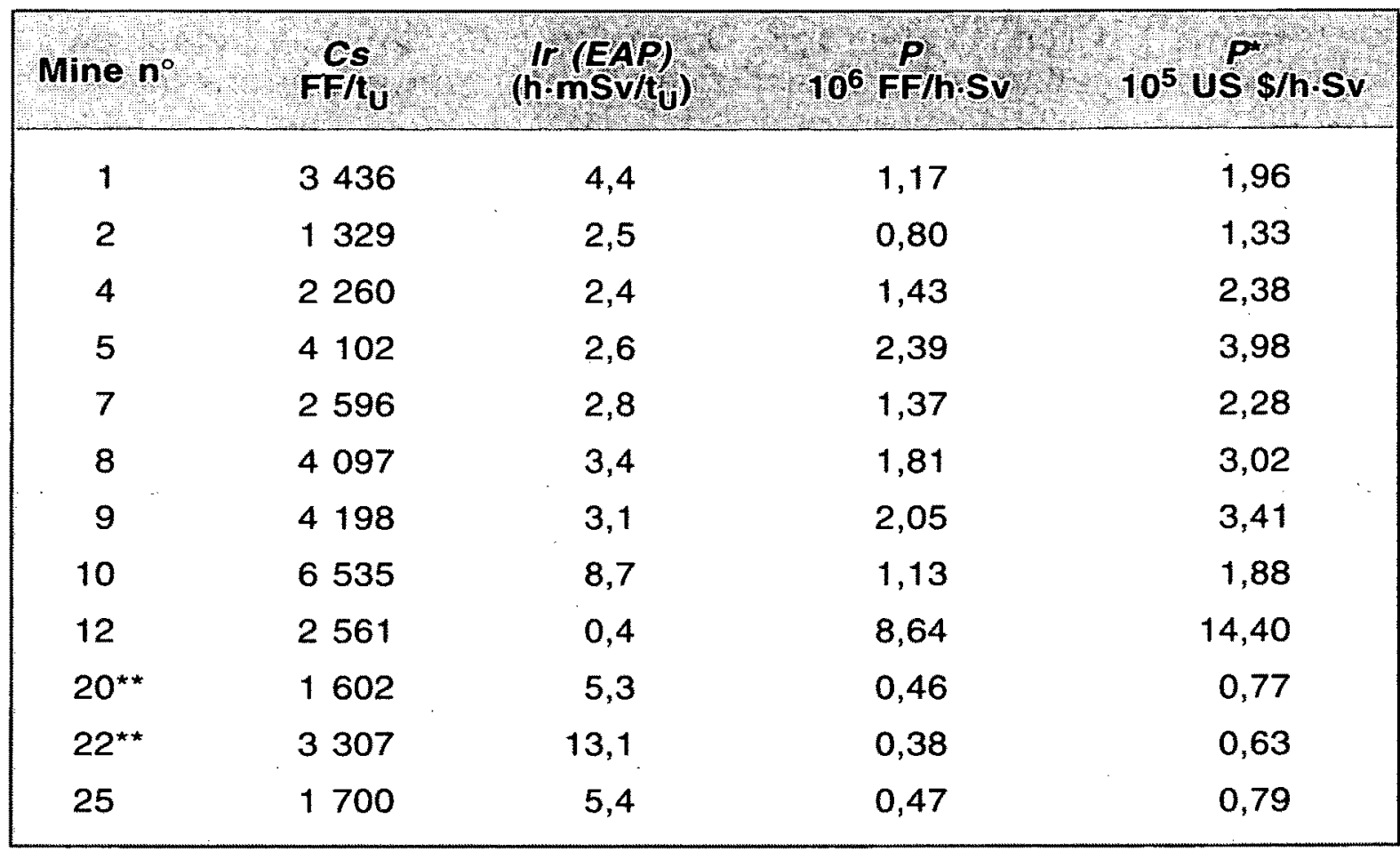

Hypothèse $K=1,5$.

* 6 FF $=1$ US $\$$.

** 1988

TABLEAU VI

Evolution des coûts spécifiques dans 9 mines souterraines de 1984 à 1989. Trend of specific costs in 9 underground mines, 1984-1989

\begin{tabular}{|c|c|c|c|c|}
\hline Mine no & Annee & $10^{6} \mathrm{FF} / \mathrm{h} \cdot \mathrm{Sv}$ & $10^{5}$ US $\$ / h \cdot S v$ & $\begin{array}{l}D_{C}(E A P) \\
(H \cdot m S v)\end{array}$ \\
\hline 1 & 1984 & 0,38 & 0,64 & 2140 \\
\hline 1 & 1985 & 0,58 & 0,96 & 1903 \\
\hline 1 & 1986 & 0,52 & 0,86 & 1907 \\
\hline 1 & 1987 & 0,61 & 1,01 & 2016 \\
\hline 1 & 1988 & 0,98 & 1,63 & 1200 \\
\hline 1 & 1989 & 1,17 & 1,98 & 1170 \\
\hline 2 & 1984 & 0,32 & 0,53 & 2473 \\
\hline 2 & 1985 & 0,49 & 0,81 & 1760 \\
\hline 2 & 1986 & 0,62 & 1,03 & 1472 \\
\hline 2 & 1987 & 0,53 & 0,88 & 1406 \\
\hline 2 & 1988 & 0,59 & 0,99 & 985 \\
\hline 2 & 1989 & 0,80 & 1,33 & 756 \\
\hline
\end{tabular}


TABLEAU VI (suite)

\begin{tabular}{|c|c|c|c|c|}
\hline Mine $n^{\circ}$ & Annee & $10^{6}$ FF/h.Sv & $10^{5}$ US $s / \mathrm{h} \cdot \mathrm{Sv}$ & $\begin{array}{r}D_{c}(E A P) \\
(H, m S v)\end{array}$ \\
\hline 4 & 1984 & 0,43 & 0,72 & 1195 \\
\hline 4 & 1985 & 0,84 & 1,34 & 598 \\
\hline 4 & 1986 & 1,19 & 1,98 & 540 \\
\hline 4 & 1987 & 1,06 & 1,77 & 730 \\
\hline 4 & 1988 & 1,40 & 2,33 & 701 \\
\hline 4 & 1989 & 1,43 & 2,38 & 570 \\
\hline 5 & 1984 & 0,39 & 0,65 & 298 \\
\hline 5 & 1985 & 0,45 & 0,75 & 260 \\
\hline 5 & 1986 & 0,43 & 0,72 & 270 \\
\hline 5 & 1987 & 0,51 & 0,86 & 319 \\
\hline 5 & 1988 & 0,36 & 0,61 & 242 \\
\hline 5 & 1989 & 2,39 & 3,98 & 66 \\
\hline 7 & 1984 & 0,52 & 0,86 & 1860 \\
\hline 7 & 1985 & 0,50 & 0,84 & 1798 \\
\hline 7 & 1986 & 0,62 & 1,03 & 1460 \\
\hline 7 & 1987 & 0,58 & 0,96 & 1942 \\
\hline 7 & 1988 & 0,53 & 0,88 & 1336 \\
\hline 7 & 1989 & 1,37 & 2,28 & 518 \\
\hline 8 & 1984 & 0,91 & 1,52 & 878 \\
\hline 8 & 1985 & 1,08 & 1,80 & 733 \\
\hline 8 & 1986 & 1,07 & 1,79 & 707 \\
\hline 8 & 1987 & 1,51 & 2,51 & 700 \\
\hline 8 & 1988 & 1,94 & 3,23 & 604 \\
\hline 8 & 1989 & 1,81 & 3,02 & 581 \\
\hline 9 & 1984 & 0,93 & 1,56 & 1690 \\
\hline 9 & 1985 & 1,36 & 2,27 & 820 \\
\hline 9 & 1986 & 1,30 & 2,16 & 861 \\
\hline 9 & 1987 & 1,56 & 2,60 & 891 \\
\hline 9 & 1988 & 1,09 & 1,81 & 990 \\
\hline 9 & 1989 & 2,05 & 3,41 & 555 \\
\hline 10 & 1984 & 0.32 & 0.53 & 258 \\
\hline 10 & $\begin{array}{l}1904 \\
1985\end{array}$ & $\begin{array}{l}0,32 \\
0,78\end{array}$ & $\begin{array}{l}0,50 \\
1,31\end{array}$ & 185 \\
\hline 10 & 1986 & 1,28 & 2,14 & 113 \\
\hline 10 & 1987 & 0,48 & 0,80 & 301 \\
\hline 10 & 1988 & 1,12 & 1,86 & 234 \\
\hline 10 & 1989 & 1,13 & 1,88 & 210 \\
\hline 12 & 1984 & 2.29 & 3.81 & 1095 \\
\hline 12 & 1985 & 2,45 & 4,09 & 1180 \\
\hline 12 & 1986 & 3,34 & 5,56 & 1080 \\
\hline 12 & 1987 & 4,57 & 7,62 & 841 \\
\hline 12 & 1988 & 6,42 & 10,70 & 625 \\
\hline 12 & 1989 & 8,64 & 14,40 & 467 \\
\hline
\end{tabular}

* NB: $6 \mathrm{FF}=1 \$$ US. 
On observe aussi (fig. 7) que les coûts marginaux de ces options observés en 1989 sont largement au-dessus de la gamme des prix de l'unité de dose collective évitée que les organisations internationales recommandent de ne pas dépasser. Ces options, qui étaient toutes acceptables du point de vue de la balance des coûts économiques et sanitaires en 1984, sauf dans le cas de la mine $n^{\circ} 12$, ne le sont plus du point de vue du principe d'optimisation, en 1989. D'autres options sont à rechercher, si elles existent, pour continuer à optimiser la protection.

\subsection{Quelques exemples de résultats de mise en cuvre des principes de l'optimisation dynamique dans le cas de mines anciennes}

Au cours des 20 dernières années, une amélioration notable de la protection radiologique s'observe dans les mines françaises [1]. L'évolution des résultats d'irradiation spécifique est montrée en figure 8 . L'amélioration constatée s'explique par différents facteurs : d'abord, l'adhésion de la direction des mines considérées à une politique optimisée selon les principes exposés plus haut, ensuite, par l'amélioration de la productivité d'extraction due au progrès des techniques minières - en particulier, amélioration de la tenue mécanique des roches permettant plus de mécanisation, diminution de la résistance aéraulique, fonçage des puits d'aérage facilité par la méthode du trépant remontant. Les besoins en air

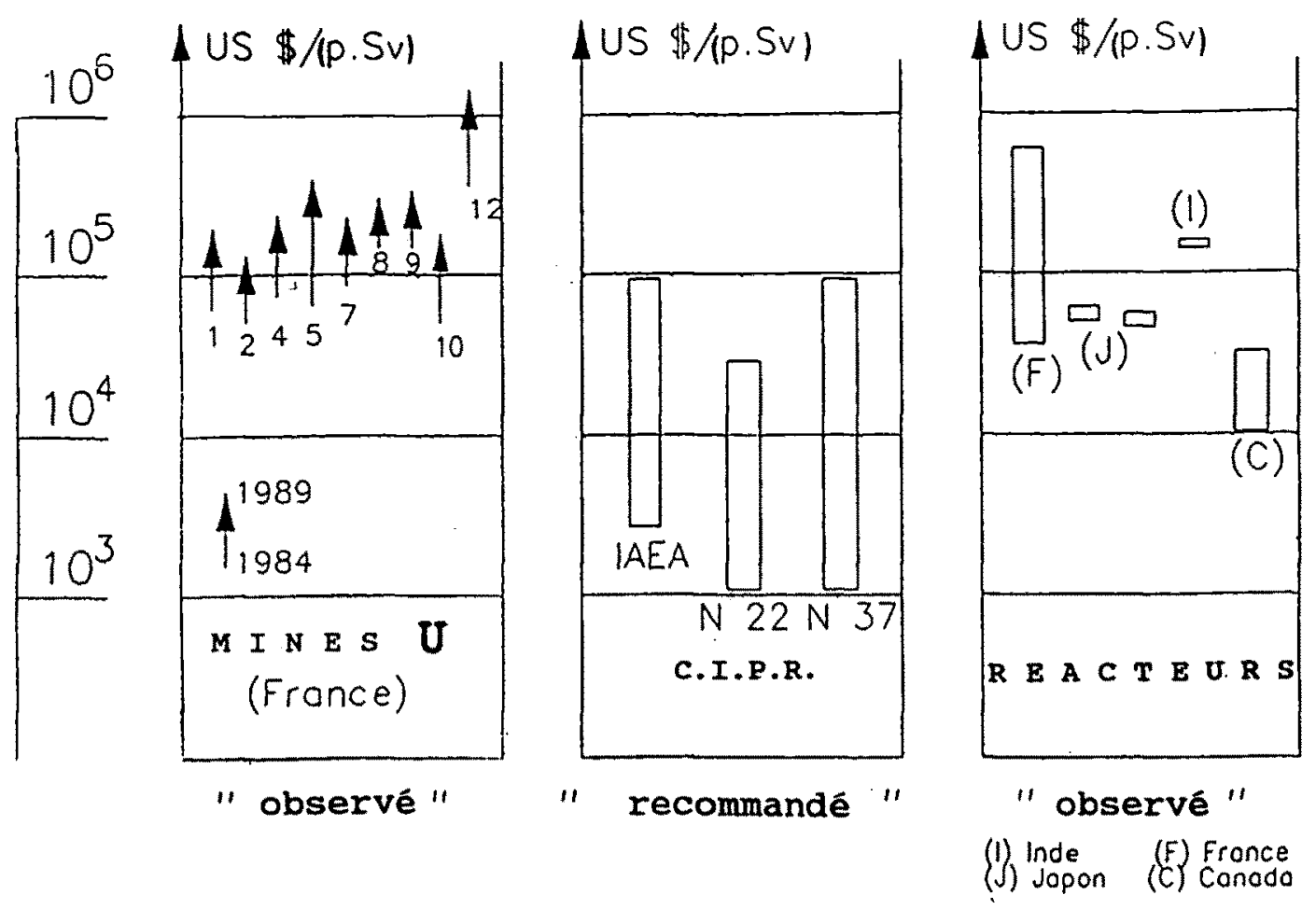

Fig. 7. - Valeurs recommandées et observées du prix de l'homme-sievert. Recommended and observed man-sievert values. 


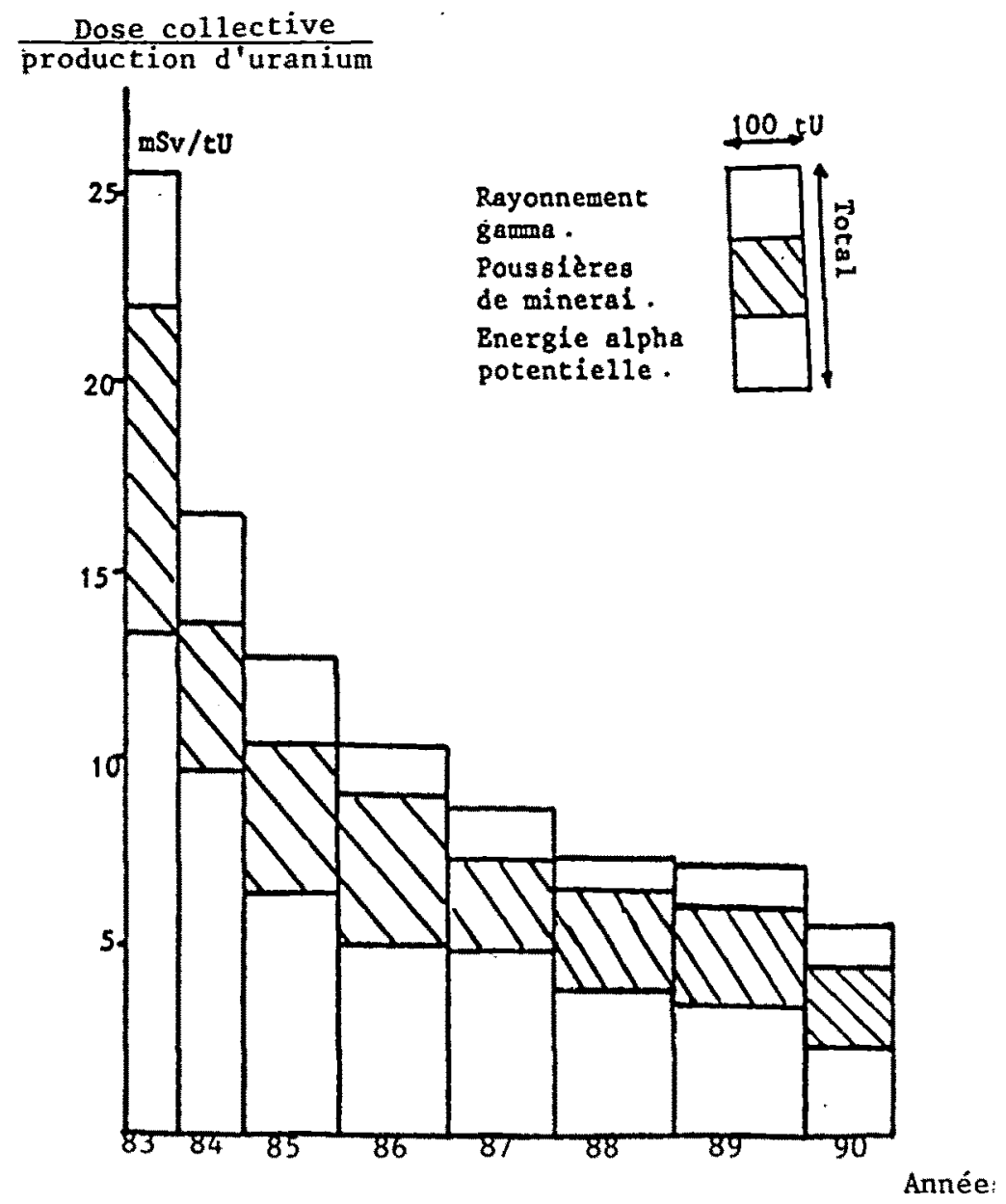

Fig. 8. - Exemple d'évolution des irradiations spécifiques depuis 1983 pour les mines souterraines de la COGEMA.

An example of the trend of collective specific exposures for COGEMA underground mines, 1983-1990.

frais imposés par la nécessité absolue de la bonne et immédiate élimination des fumées de diésel sont souvent supérieurs à ceux qui seraient imposés par l'intensité locale des sources de radon 222 dans les chantiers d'abattage, ce dont la protection radiologique a profité dans un premier temps. Les méthodes minières, elles-mêmes, ont évolué dans le bon sens, en particulier, la mise en place des méthodes d'exploitation par tranches descendantes sous dalles bétonnées permettent souvent de maintenir les chantiers d'abattage en aérage primaire, ce qui leur permet d'échapper aux aléas de l'aérage secondaire.

Les améliorations de la protection radiologique s'expliquent, enfin, par la mise en œuvre de l'optimisation dynamique [6]. II n'y a pas d'optimisation possible sans une comptabilisation rigoureuse et crédible des doses individuelles. Dans les débuts, la seule stratégie de dosimétrie relative aux risques d'exposition interne disponible était la dosimétrie d'ambiance, c'est-à-dire une dosimétrie qui n'est ni rigoureuse, ni crédible, en mine 
souterraine tout particulièrement. C'est pourquoi, dans un premier temps, un effort prioritaire a été consacré par le CRPM au développement des moyens d'une dosimétrie individuelle concernant les risques radiologiques miniers, non seulement le risque d'exposition externe mais aussi les risques d'exposition interne due aux descendants à vie courte du radon et aux émetteurs alpha à vie longue. Cet effort a permis l'instauration généralisée de la dosimétrie individuelle de tous les personnels du fond des mines francaises dès le $1^{\text {er }}$ janvier 1983.

Les principes et les méthodes de suppression des gaspillages, plus haut exposés, ont alors pu être progressivement mis en œuvre [9].

Comme il a été indiqué è 5.3, des options de réductions supplémentaires de la dose collective, possibles techniquement, vont commencer à devenir, pour ces mines, des options qui dépassent l'optimum de radioprotection et donc non recommandables.

\section{Conclusion}

On peut encore améliorer la qualité de la protection radiologique dans les mines. En premier lieu, sont concernées les mines qui ne respectent pas les réglementations actuelles issues des publications CIPR 26 et 47 , [2-3]. Les résultats obtenus sur un grand nombre de mines montrent qu'il est possible techniquement et économiquement, qu'il s'agisse de mines de substances radioactives ou non radioactives, de respecter la limite individuelle, $50 \mathrm{mSv} / \mathrm{an}$, et d'optirniser le niveau de protection collective à des prix de l'homme-sievert évité raisonnables.

En ce qui concerne les mines qui respectent déjà ces réglementations, les améliorations, si elles sont encore possibles, sont à rechercher, non par la limitation plus sévère des doses individuelles, mais dans le cadre du principe d'optimisation, voie réaliste compte-tenu des progrès faits dans la compréhension des mécanismes de formation de la dose collective relative aux différentes voies d'exposition et des progrès des techniques et des méthodes minières. Toutefois, on atteint relativement vite, dans les mines en exploitation, le moment où le prix de la dose collective marginale évitée devient excessif par rapport à sa valeur sanitaire estimée.

Dans les mines futures, l'introduction, dès le moment de la conception de l'exploitation à venir, du principe d'optimisation de la protection radiologique permet, souvent, d'aller plus loin en ce qui concerne l'irradiation spécifique et le risque individuel moyen annuel.

L'augmentation de la sévérité des limites individuelles d'acceptabilité et de classement des travailleurs exposés n'apparaît pas à recommander à cause de toute une série d'effets pervers qui finiraient par porter le discrédit sur l'ensemble du système de gestion des risques radiologiques. La raison fondamentale est, en fait, que le domaine des doses profes- 
sionnelles à gérer par l'industriel finit par être à l'intérieur du domaine des doses reçues par les personnes du public pour les mêmes radionucléides (descendants à vie courte des radons 220 et 222) et les mêmes voies d'exposition (inhalation), et pour lequel la CIPR recommande la non intervention (exemption), c'est-à-dire l'absence de gestion.

\section{RÉFÉRENCES}

[1] BERNHARD S., KRAEMER G., ZETTWOOG P. - La radioprotection dans les mines et usines de minerai d'uranium françaises. Radioprotection, 1991, 26 (2) 329-349.

[2] COMMISSION INTERNATIONALE DE PROTECTION RADIOLOGIQUE (CIPR) Recommandations... (Publication CIPR 26). Oxford : Pergamon press, 1978.

[3] COMMISSION INTERNATIONALE DE PROTECTION RADIOLOGIQUE (CIPR) Radiation protection of workers in mines. (ICRP publication 47). Oxford : Pergamon press, 1986.

[4] COMMISSION INTERNATIONALE DE PROTECTION RADIOLOGIQUE (CIPR) Recommendations... (ICRP publication 60). Oxford : Pergamon press, 1991.

[5] COMMISSION INTERNATIONALE DE PROTECTION RADIOLOGIQUE (CIPR) Annual limits on intake of radionuclides by workers based on the 1990 recommendations. (ICRP publication 61). Oxford : Pergamon press, 1991.

[6] FOURCADE N., ZETTWOOG P. - A dynamic approach to the implementation of the optimization principles in uranium mines. In : Occupational radiation safety in mining, Toronto, October, 14-18, 1984 (Stocker H., Ed.). Toronto : Canadian Nuclear Association, 1985, Vol. 1, 57-71.

[7] FRANCE. INDUSTRIE ET AMENAGEMENT DU TERRITOIRE (Ministère) - Décret $n^{\circ}$ 89-502 du 13 juillet 1989 complétant le règlement général des industries extractives institué par le décret $n^{\circ} 80-331$ du 7 mai 1980 modifié et Circulaire du 13 juillet 1989 relative à l'application du décret $n^{\circ} 89-502$ du 13 juillet 1989 complétant le règlement général des industries extractives (rayonnements ionisants RI-1. C). J.O.R.F., 20 juillet 1989 , 9053-9058; 9073-9075.

[8] MULLER-KAHLE E. - The international uranium situation in view of the new radiation dose limit proposal. In : Uranium and nuclear energy 1988, London, September 7-9, 1988. Londres : Uranium institute, 1989.

[9] OUDIZ A., LOMBARD J., ZETTWOOG P. - Un exemple d'optimisation de la protection radiologique des mineurs d'uranium. Radioprotection, 1984, 19 (1), 23-35.

[10] ZETTWOOG P. - Modélisation des irradiations spécifiques dans les mines d'uranium. Séminaire, Vassivière, 3-4 octobre 1989. 